\title{
Dynamic Behaviors and Energy Transition Mechanism of Droplets Impacting on Hydrophobic Surfaces
}

\author{
Qiaogao Huang, Ya Zhang, and Guang Pan \\ School of Marine Science and Technology, Northwestern Polytechnical University, Xian, Shaanxi 710072, China \\ Correspondence should be addressed to Qiaogao Huang; huangqiaogao@163.com
}

Received 23 December 2015; Accepted 3 April 2016

Academic Editor: Luisa Di Paola

Copyright (C) 2016 Qiaogao Huang et al. This is an open access article distributed under the Creative Commons Attribution License, which permits unrestricted use, distribution, and reproduction in any medium, provided the original work is properly cited.

\begin{abstract}
The wettability of hydrophobic surfaces and the dynamic behaviors of droplets impacting on hydrophobic surfaces are simulated using a lattice Boltzmann method, and the condition for the rebound phenomenon of droplets impacting on solid surfaces is analyzed. The results show that there is a linear relationship between the intrinsic contact angle and the interaction strength of fluid-wall particles. For hydrophobic surfaces with the same intrinsic contact angle, the micromorphology can increase the surface hydrophobicity, especially the hierarchical micromorphology. The dynamic behaviors of droplets impacting on solid surfaces are affected by the wettability. The surface hydrophobicity is stronger, and the rebound phenomenon occurs easier. If the droplet's kinetic energy is greater than the sum of the surface energy and the minimum conversion gravitational potential energy when the spreading and shrinking finish, the rebound phenomenon will occur. As the hydrophobic surface's viscous dissipation is much smaller than the hydrophilic surface's, the droplet still has high kinetic energy after the spreading and shrinking, which is advantageous to rebound for droplets.
\end{abstract}

\section{Introduction}

Recently, more and more researches demonstrate that hydrophobic surface has a good applied prospect in aspects of fluid drag reduction, flow noise reduction, anticontamination, anticorrosion, and so forth [1-3]. For instance, it is used as drag reduction and anticontamination coating of pipe's inner surface and ship's hull, as self-cleaning coating of exterior walls and satellite receivers, and as anti-icing and antifrosting coating of components of aeronautics and astronautics, which, with no exception, are related to the movement of droplets on hydrophobic surfaces, especially the dynamic behaviors of droplets impacting on hydrophobic surfaces. Davidson [4] investigated droplets impinging on solid surfaces using a boundary integral method. Pasandideh-Fard et al. [5] simulated the solidification of molten metal droplets on both horizontal and inclined stainless steel surfaces with VOF (Volume of Fluid) and continuous surface tension model. Fujimoto et al. [6] also employed VOF to study the effect of the impact angle on the deformation behavior of droplets. Although several computational models for studying the dynamic behaviors of droplet impacting on solid surface have been developed by researchers, the difficulty of this issue still relies on how to accurately track the position of the free surface. Lattice Boltzmann method, as a new computational fluid method, operates on both mesoscopic and macroscopic levels successfully. In particular, when things come to the numerical simulation of multiphase and multicomponent fluid flows, Shan-Chen model of lattice Boltzmann method can realize thermodynamic phase transition easily with an appropriate potential function [7, 8]. Huang et al. [9] studied the movement of a droplet inside a grooved channel using lattice Boltzmann method. Shi et al. [10] simulated numerically the droplet motion driven by Marangoni effect, which is induced by surface tension gradient on solid-liquid interface with LBM. However, their main concern was the movement of droplets on solid surfaces, and little attention was paid on the hydrodynamic behaviors and energy transition mechanism of droplets impacting on solid surfaces. Hence, this paper investigates the dynamic behaviors of droplets impacting on solid surfaces with different wettability using LBM and discusses the occurring condition of droplets bouncing when the solid surface gets hit from the view of energy. 


\section{Numerical Method}

2.1. Lattice Boltzmann Method. Lattice Boltzmann method, based on mesoscopic kinetic models, has developed into an alternative and promising numerical scheme for simulating fluid flows in recent years. Lattice Boltzmann method, presented in this paper, is based on the streaming and collision of discrete particle density distribution function in the fixed lattice point, and the corresponding evolution function is expressed as [11]

$$
\begin{aligned}
f_{\alpha} & \left(\mathbf{x}+\mathbf{e}_{\alpha} \delta_{t}, t+\delta_{t}\right)-f_{\alpha}(\mathbf{x}, t) \\
& =-\frac{1}{\tau}\left[f_{\alpha}(\mathbf{x}, t)-f_{\alpha}^{(\mathrm{eq})}(\mathbf{x}, t)\right],
\end{aligned}
$$

where $f_{\alpha}(\mathbf{x}, t)$ is the particle density distribution function, which depends on position $\mathbf{x}$, the particle discrete velocity $\mathbf{e}_{\alpha}$, and time $t, f_{\alpha}^{(\mathrm{eq})}(\mathbf{x}, t)$ is the equilibrium distribution function, and $\tau$ is the dimensionless relaxation time related to the hydrodynamics viscosity.

The left-side of lattice Boltzmann equation represents the streaming of particles, while the right-side represents the collision process, which makes $f$ heading for the equilibrium distribution $f^{(\mathrm{eq})}$. In D2Q9 model [11], the equilibrium distribution $f^{(\mathrm{eq})}$ is given by

$$
f_{\alpha}^{(\mathrm{eq})}(\mathbf{x}, t)=\rho \omega_{\alpha}\left[1+\frac{\mathbf{e}_{\alpha} \cdot \mathbf{u}}{c_{s}^{2}}+\frac{\left(\mathbf{e}_{\alpha} \cdot \mathbf{u}\right)^{2}}{2 c_{s}^{4}}-\frac{u^{2}}{2 c_{s}^{2}}\right] .
$$

The discrete velocities are set as

$$
\begin{aligned}
& {\left[\mathbf{e}_{0}, \mathbf{e}_{1}, \mathbf{e}_{2}, \mathbf{e}_{3}, \mathbf{e}_{4}, \mathbf{e}_{5}, \mathbf{e}_{6}, \mathbf{e}_{7}, \mathbf{e}_{8}\right]} \\
& \quad=\left[\begin{array}{ccccccccc}
0 & 1 & 0 & -1 & 0 & 1 & -1 & -1 & 1 \\
0 & 0 & 1 & 0 & -1 & 1 & 1 & -1 & -1
\end{array}\right] c,
\end{aligned}
$$

where $c=\delta_{x} / \delta_{t}$ is lattice velocity; $\delta_{x}$ and $\delta_{t}$ are the lattice length and the time step, respectively; $c_{s}=c / \sqrt{3}$ is the sound speed in the discrete model, and the weighting factors depend on the link angle $\left(\omega_{\alpha}=4 / 9(\alpha=0) ; \omega_{\alpha}=1 / 9(\alpha=1,2,3,4)\right.$; $\left.\omega_{\alpha}=1 / 36(\alpha=5,6,7,8)\right)$. follows:

The macroscopic density and velocity are defined as

$$
\begin{aligned}
& \rho=\sum_{\alpha} f_{\alpha}, \\
& \mathbf{u}=\frac{1}{\rho} \sum_{\alpha} \vec{e}_{\alpha} f_{\alpha} .
\end{aligned}
$$

The macroscopic pressure is given by $p=\rho c_{s}^{2}$ directly.

2.2. Shan-Chen Model. To introduce intermolecular forces between microscopic particles, Shan and Chen proposed a pseudopotential model $[12,13]$, which easily coupled microforces, the dominant role of microflow problems. The model reflects the physical nature of fluid dynamics and broadens the application of lattice Boltzmann method in range of simulation of microflow on complex surface with wettability.
In Shan-Chen model, fluid particles at site of $\mathbf{x}$ receive forces from nearest neighbor fluid particles and solid walls, which are written in the following form, respectively:

$$
\begin{aligned}
& \mathbf{F}_{\text {int }}(\mathbf{x}, t)=-G \psi(\mathbf{x}, t) \sum_{\alpha=1}^{8} w_{\alpha} \psi\left(\mathbf{x}+\mathbf{e}_{\alpha} \delta_{t}, t\right) \mathbf{e}_{\alpha}, \\
& \mathbf{F}_{\text {ads }}(\mathbf{x}, t)=-G_{s} \psi(\mathbf{x}, t) \sum_{\alpha=1}^{8} w_{\alpha} \operatorname{swi}\left(\mathbf{x}+\mathbf{e}_{\alpha} \delta_{t}\right) \mathbf{e}_{\alpha} .
\end{aligned}
$$

$G$ in (5) is the interaction strength, and $G_{s}$ in (6) is called adsorption parameter, representing the strength of the force contributed by solid. Varying the $G_{s}$ parameter allows simulation of different solid surface wettability, where swi is a "switch" that takes on value one if the site at $\mathbf{x}+\mathbf{e}_{\alpha} \delta_{t}$ is a solid and is zero otherwise. $w_{\alpha}$ is the same directiondependent weighting factor used before. $\psi(\mathbf{x}, t)$ is the interaction potential function, which must be monotonically increasing and bounded. Therefore, the equation used in this paper is expressed as $\psi(\mathbf{x}, t)=\rho_{0}\left[1-\exp \left(-\rho / \rho_{0}\right)\right]$.

In Shan-Chen model, the effect of interparticle forces is incorporated into the equilibrium distribution function $\mathbf{f}^{\mathrm{eq}}$ by shifting the equilibrium velocity $\mathbf{u}^{*}$ :

$$
\begin{gathered}
\rho(\mathbf{x}, t)=\sum_{\alpha=0}^{8} f_{\alpha}(\mathbf{x}, t), \\
\rho(\mathbf{x}, t) \mathbf{u}^{*}(\mathbf{x}, t)=\sum_{\alpha=0}^{8} \mathbf{e}_{\alpha} f_{\alpha}(\mathbf{x}, t)+\tau\left(\mathbf{F}_{\mathrm{int}}+\mathbf{F}_{\mathrm{ads}}\right) .
\end{gathered}
$$

The equation of state is given by

$$
p=\rho c_{s}^{2}+\frac{G}{6} \psi^{2}
$$

\section{Numerical Simulation of the Wettability of Hydrophobic Surfaces}

A key parameter for characterizing the wettability of hydrophobic surface is the apparent contact angle, which is mainly determined by material properties and micromorphology. This provides a new research route: first, determine the relationship between the adsorption parameter $G_{s}$ and the intrinsic contact angle at a specific interaction strength $G$ by simulating the contact angle of a smooth solid surface; then construct microtopography on the surface and simulate the apparent contact angle of real solid surface.

3.1. Determining the Intrinsic Contact Angle. The intrinsic contact angle of the smooth solid surface is adjusted by changing the adsorption parameter $G_{s}$ for a specific interaction strength $G=-5.8$. The computational domain is a rectangular space of $500 \times 500$ lattice units, and a lattice units is approximately $1.2 \mu \mathrm{m}$ in physical scale. Dimensionless time $\tau=1.0$, and gravity acceleration $g=0.5 \times 10^{-6}$. Periodic scheme is used in the left and right boundaries, while no-slip bounce back scheme is used in the top and bottom boundaries. A circular liquid droplet with a diameter of 150 is placed in the middle of the bottom wall. 


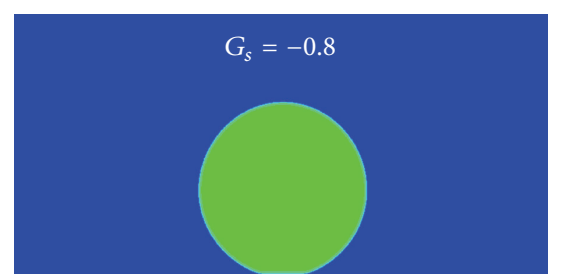

(a) $\mathrm{CA}=159.3^{\circ}$

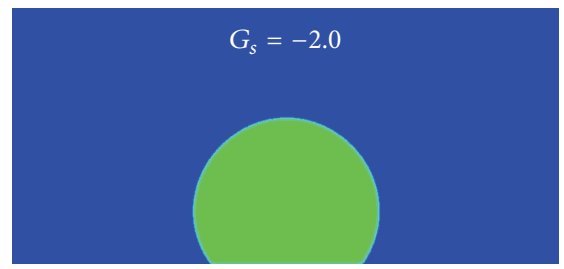

(d) $\mathrm{CA}=120.5^{\circ}$

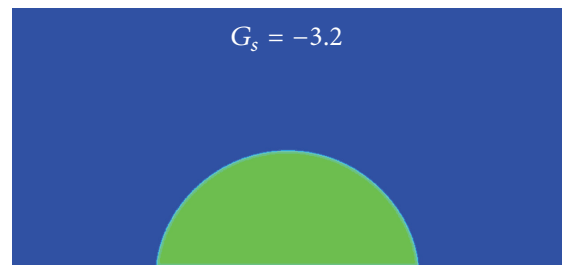

(g) $\mathrm{CA}=81.6^{\circ}$

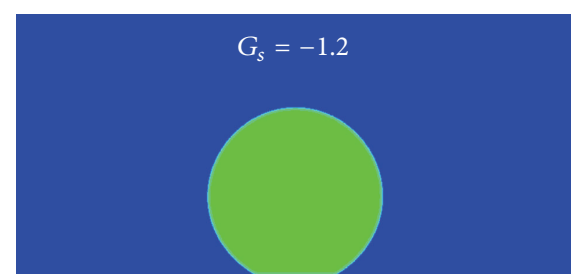

(b) $\mathrm{CA}=145.4^{\circ}$

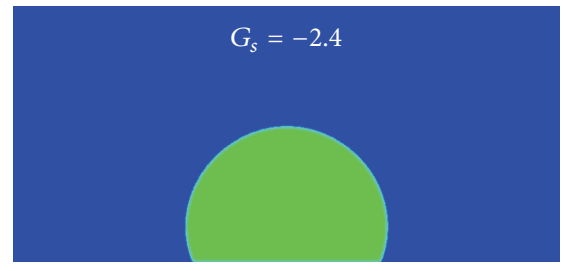

(e) $\mathrm{CA}=108.9^{\circ}$

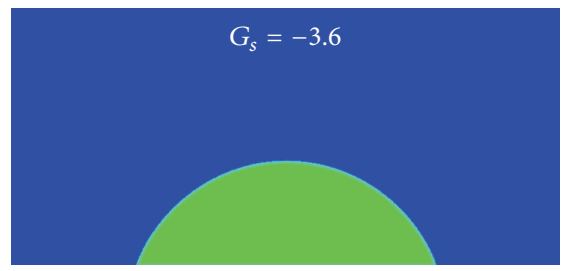

(h) $\mathrm{CA}=68.1^{\circ}$

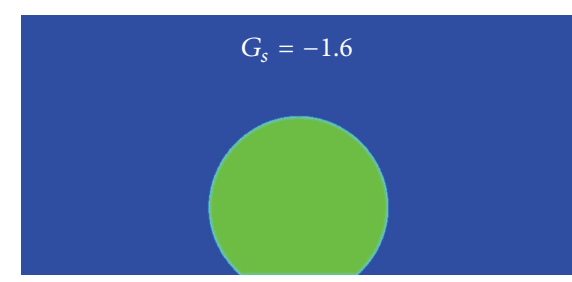

(c) $\mathrm{CA}=132.7^{\circ}$

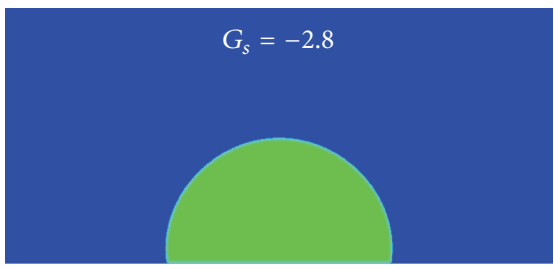

(f) $\mathrm{CA}=96.8^{\circ}$

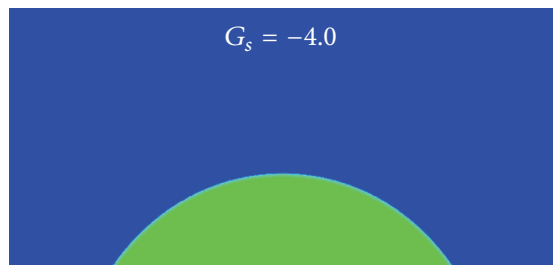

(i) $\mathrm{CA}=56.2^{\circ}$

FIGURE 1: Droplet shape and intrinsic contact angle of smooth surfaces under different adsorption parameter.

By the different forces from the solid wall, the fluid drops show different shapes when the system reaches equilibrium. Shapes and contact angles of droplets for different absorption coefficients are shown in Figure 1. The result of fitting $\theta$ and $\left|G_{s}\right|$ in Figure 2 illustrates that there is a linear relationship between $\theta$ and $\left|G_{s}\right|$, and $\theta$ decreases with the increase of $\left|G_{s}\right|$. The fitting result is expressed as the linear equation

$$
\theta=-32.09\left|G_{s}\right|+184.74 \text {. }
$$

According to (9), for $2.952<\left|G_{s}\right|<5.757$, the solid material is hydrophilic with $0^{\circ}<\theta<90^{\circ}$; for $0.148<\left|G_{s}\right|<$ 2.952 , the solid material is hydrophobic with $90^{\circ}<\theta<180^{\circ}$.

3.2. Determining the Apparent Contact Angle. In order to simulate the apparent contact angle of real solid surface, we need to build microtopography on the smooth solid surfaces that have specific material properties. In the simulation, the $2 \mathrm{D}$ square cylinders represent the rough elements on the hydrophobic surface. The cylinders are defined by parameters of the column, width $a$, spacing $b$, and height $h$. When computations are converged, we obtain the droplet shape and the apparent contact angle of different hydrophobic surfaces, which are shown in Figure 3. Simulations in Figure 3 are based on the same absorption parameter, which means these surfaces share the same material properties. According to the figure, the contact angle of the droplet on the smooth hydrophobic surface is $108.9^{\circ}$, indicating a relatively weak hydrophobicity. However, after adding a rough structure on the surface, its hydrophobicity is improved significantly.

For droplets placed on composite surfaces shown in Figure 3, when the gas-liquid interfacial fraction $f_{v}=b /(a+$

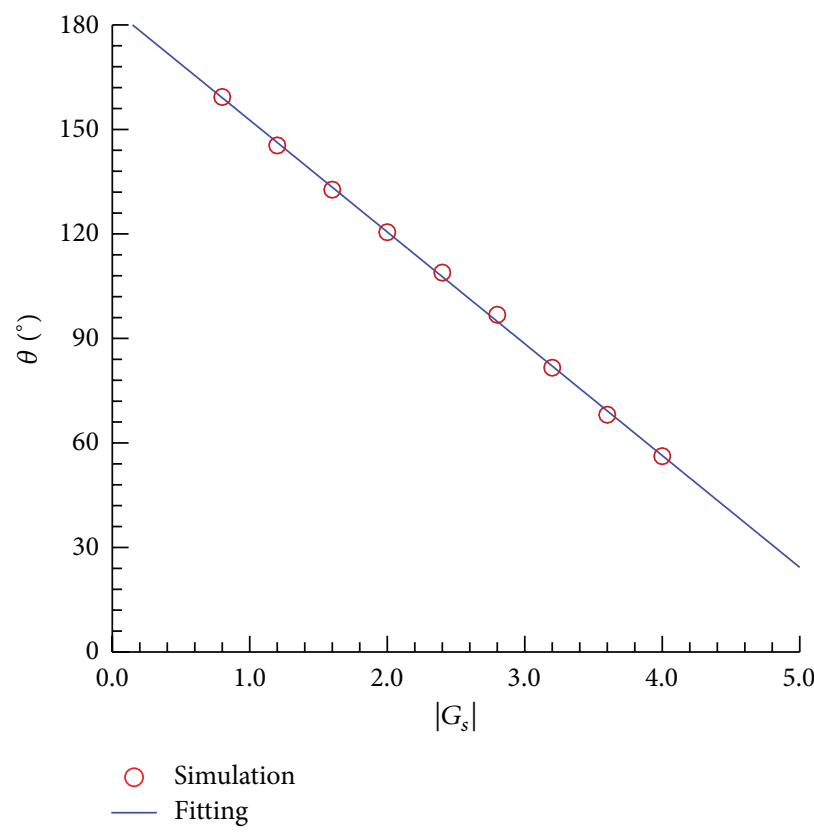

FIGURE 2: The relationship between intrinsic contact angle and adsorption parameter.

b) $=0.333$, the apparent contact angle is $123.1^{\circ}$; when $f_{v}=$ 0.667 , the contact angle is $123.1^{\circ}$; when the surface is decorated by hierarchical micromorphology, the contact angle is $151.8^{\circ}$, superhydrophobicity. The results validate the previous theory obtained by experimental studies that surfaces with 


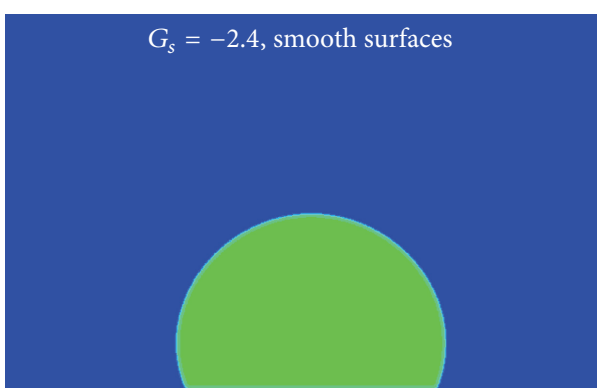

(a) $\mathrm{CA}=108.9^{\circ}$

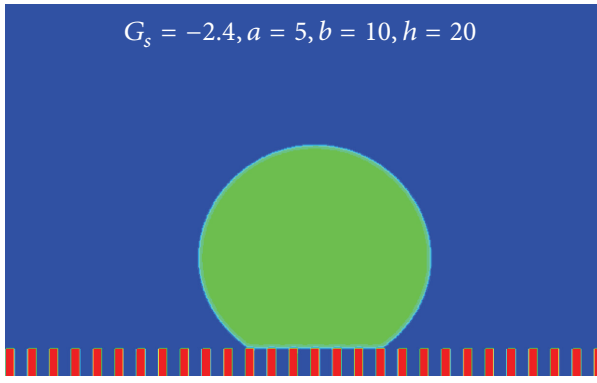

(c) $\mathrm{CA}=139.7^{\circ}$

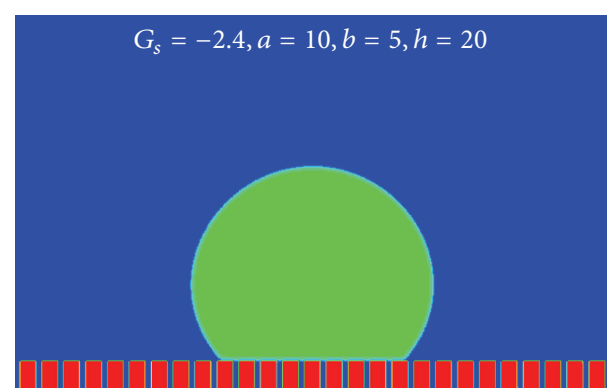

(b) $\mathrm{CA}=123.1^{\circ}$

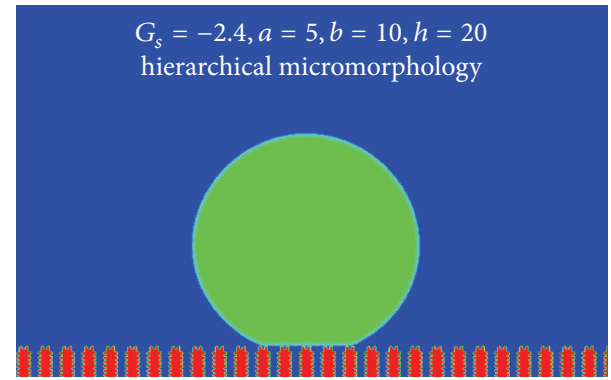

(d) $\mathrm{CA}=151.8^{\circ}$

FIGURE 3: Droplet shape and apparent contact angle of different hydrophobic surfaces.

TABLE 1: The property of hydrophilic and hydrophobic surface.

\begin{tabular}{lccccc}
\hline & $G_{s}$ & $a$ & $b$ & $h$ & Apparent contact angle \\
\hline Hydrophobic surface & -4.0 & 10 & 10 & 20 & $42.5^{\circ}$ \\
Hydrophobic surface A & -2.8 & 10 & 10 & 20 & $119.3^{\circ}$ \\
Hydrophobic surface B & -2.4 & 10 & 10 & 20 & $138.2^{\circ}$ \\
Hydrophobic surface C & -2.4 & 5 & 10 & 20 & $151.8^{\circ}$ (hierarchical micromorphology) \\
\hline
\end{tabular}

hierarchical micromorphology are more hydrophobic and explain why lotus leaf is superhydrophobic-lotus leaf has micron-sized mastoids that are decorated with nanoscale waxy crystals.

As the apparent contact angles are $123.3^{\circ}$ and $140.8^{\circ}$, respectively, for gas-liquid interfacial fractions $f_{v}=0.333$ and $f_{v}=0.667$, according to Cassie-Baxter's wettability state equation $\cos \theta_{\mathrm{CB}}=\left(1-f_{v}\right) \cos \theta_{Y}-f_{v}$, we can get the value of intrinsic contact angle $\theta_{Y}=108.9^{\circ}$ easily which is in good agreement with the analytic result. It is proved that Shan-Chen model of lattice Boltzmann method is capable of considering both material properties and microtopography simultaneously, so it is very suitable for studying problems of microflows on complex wettability surfaces.

\section{The Numerical Simulation of the Dynamic Behaviors of Droplets Impacting on Hydrophobic Surface}

The two dimensionless values used to describe the dynamic behaviors of droplets impacting are Weber number We and Reynolds Re. The former one can be thought of a measure of the relative importance of the fluid's inertia compared to its surface tension, while the latter one quantifies the relative importance of the fluid's inertia to its viscous forces. The two numbers are defined blow:

$$
\begin{gathered}
\mathrm{We}=\frac{\rho D_{0} V_{0}^{2}}{\sigma}, \\
\operatorname{Re}=\frac{D_{0} V_{0}}{\nu_{l}},
\end{gathered}
$$

where $\rho$ is the density of droplet, $D_{0}$ is the initial droplet diameter, $V_{0}$ is the droplet impacting velocity, $\sigma$ is the surface tension, and $\nu_{l}$ is the kinematic viscosity of the fluid.

The computational domain is still a rectangular space of $500 \times 500$ lattice units. Periodic scheme is used in the left and right boundaries, while standard bounce back scheme is used in the top and bottom fixed boundaries. A circular liquid droplet with a diameter of 150 and an initial downward velocity is placed in the middle of the bottom wall. The droplet is subjected to a uniform vertical downward force field. Because this study focused on the impact of the wettability of solid surface on the dynamic behaviors of droplets, We and Re are set to constants: $\mathrm{We}=5.0$ and $\mathrm{Re}=36$. The wettability is adjusted by changing the absorption parameter and geometric parameters of microtopography. The study objects are typical hydrophilic surfaces and hydrophobic surfaces, whose properties are shown in Table 1. 

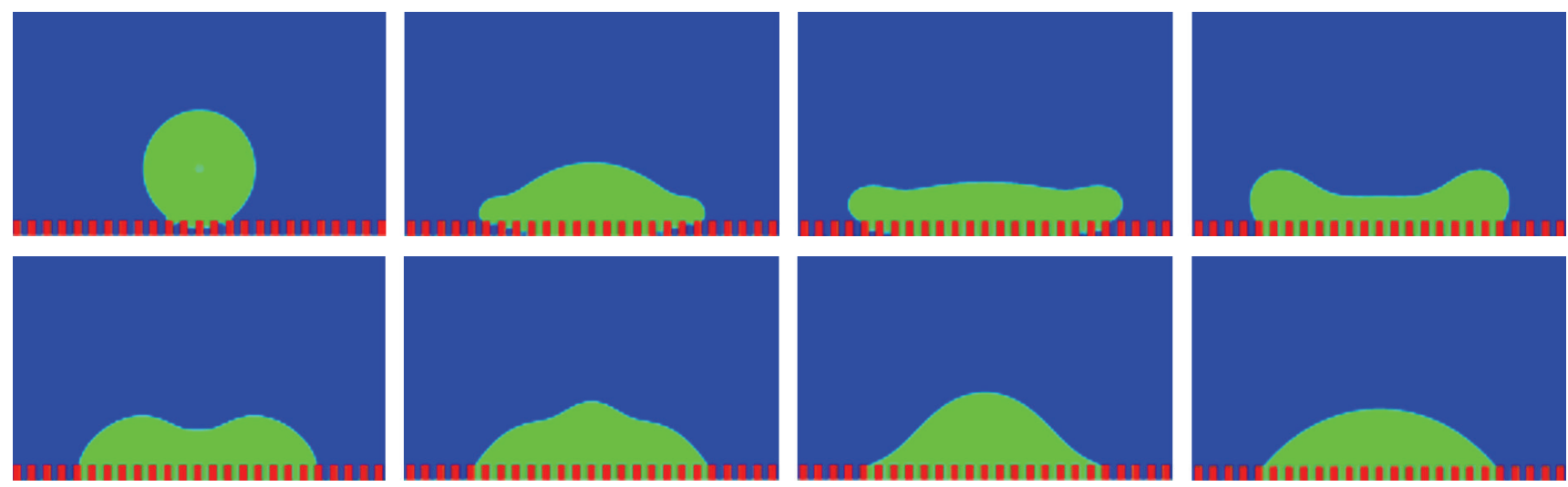

FIgURE 4: The dynamic behaviors of droplet impacting on hydrophilic surface $\left(\mathrm{CA}=42.5^{\circ}\right)$.
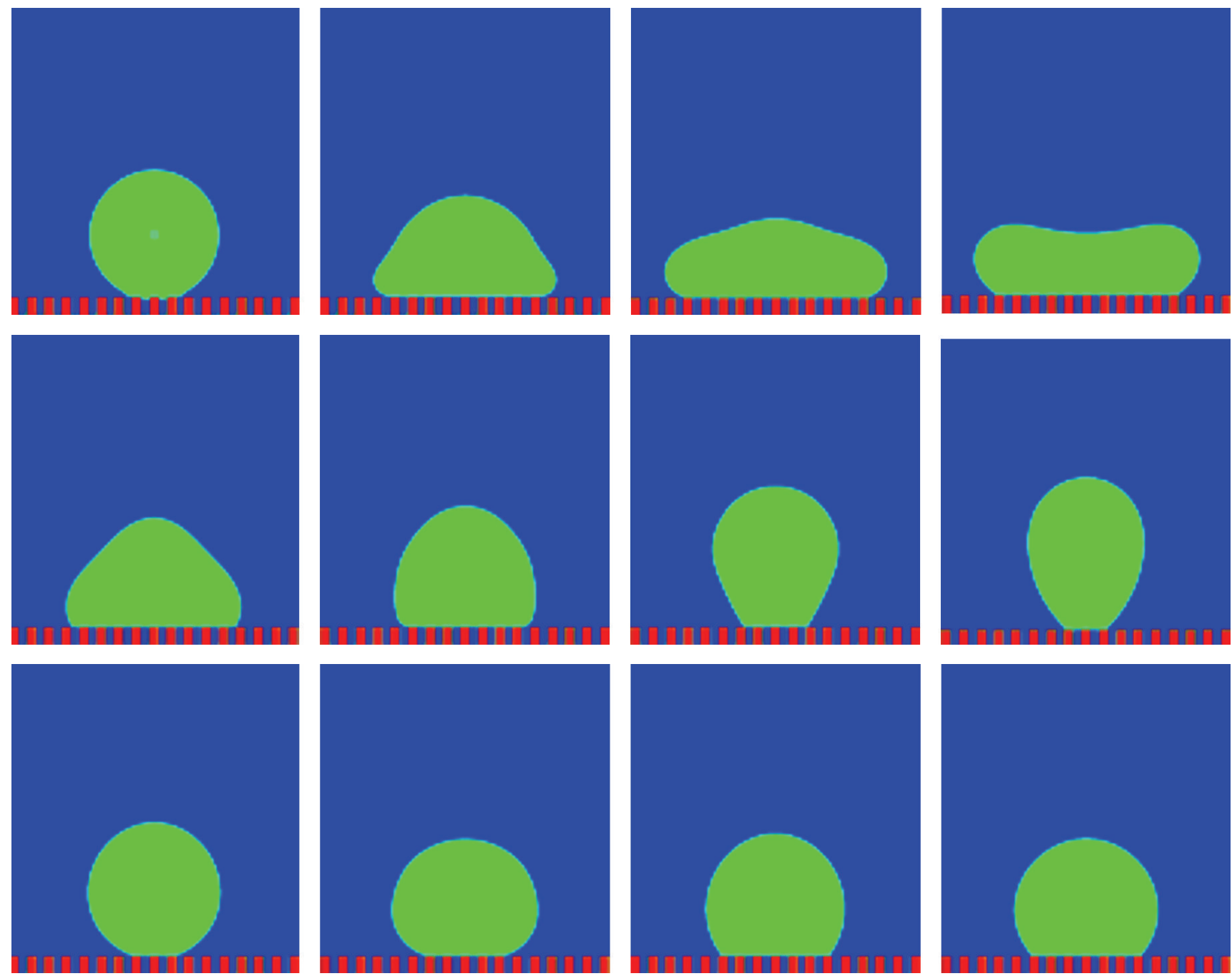

Figure 5: The dynamic behaviors of droplet impacting on hydrophobic surface $\left(\mathrm{CA}=119.3^{\circ}\right)$.

Figures 4-7 show the dynamic process of droplets impacting on different solid surfaces, respectively. It can be found from the five figures that droplets on different solid surfaces all experienced the two processes: spreading and shrinking.
After the droplet is in contact with the solid surface, fluid spreads along the radius under the action of inertial force, that is, spreading; when the droplet spreads to its maximum, fluid shrinks along the radius under the action of surface tension, 

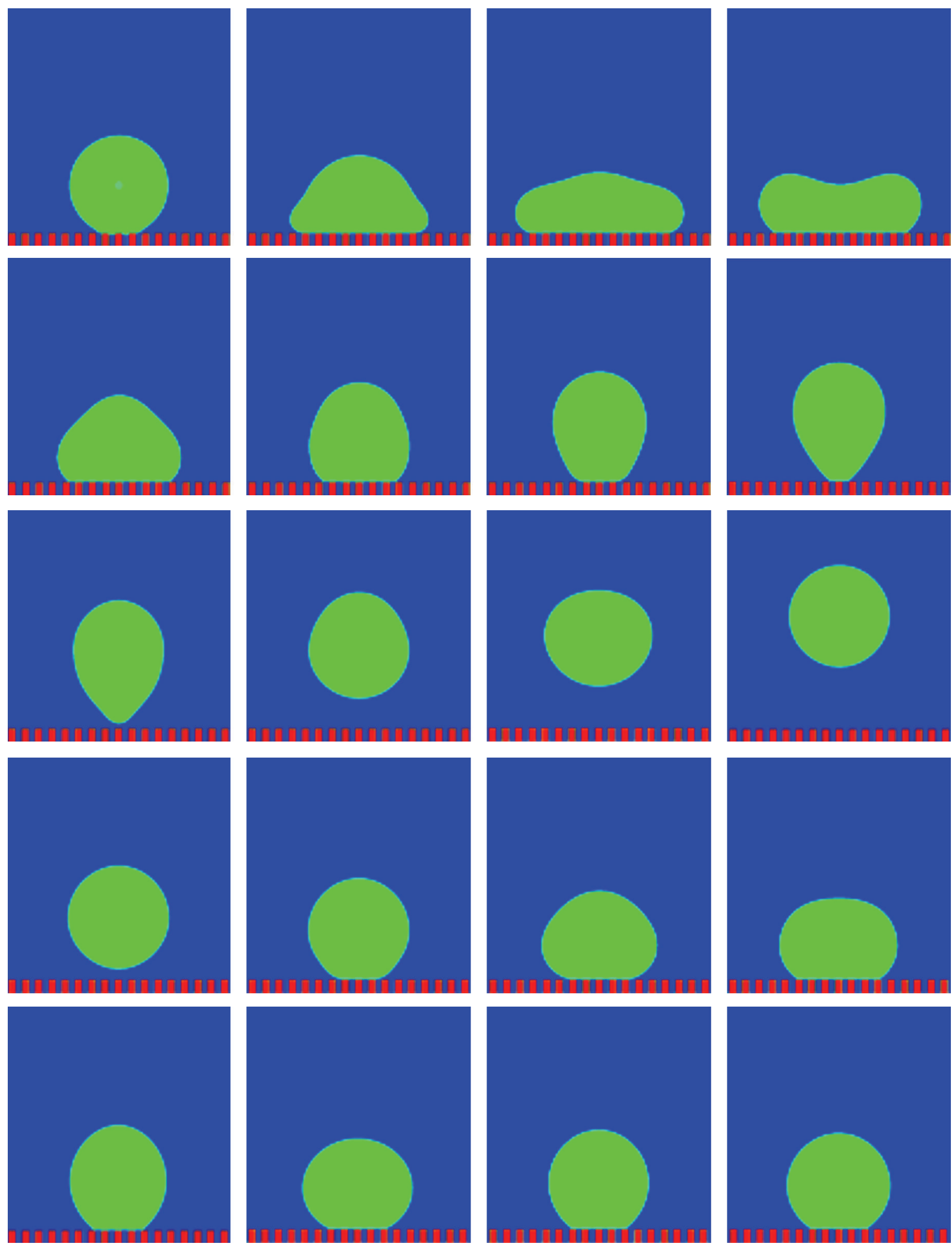

FIGURE 6: The dynamic behaviors of droplet impacting on hydrophobic surface $\left(\mathrm{CA}=138.2^{\circ}\right)$. 

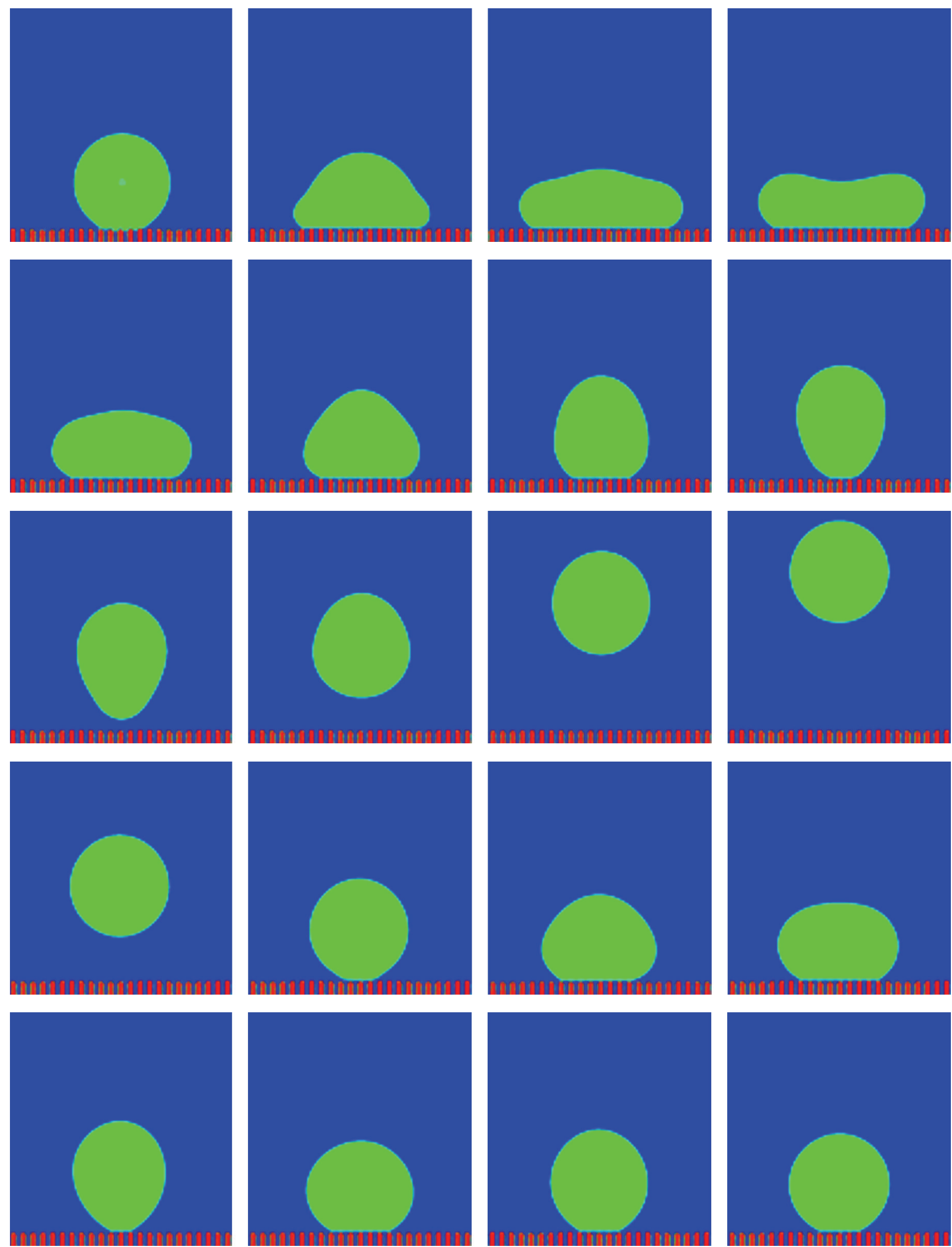

FIGURE 7: The dynamic behaviors of droplet impacting on hydrophobic surface $\left(\mathrm{CA}=151.8^{\circ}\right)$. 


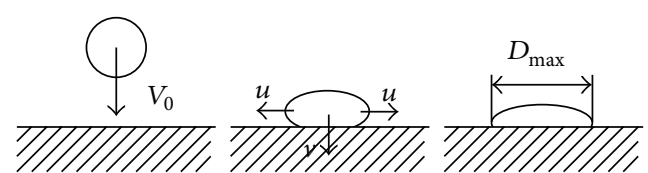

(a)
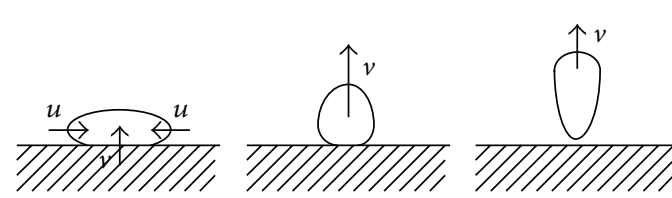

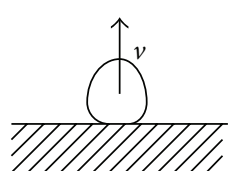

(b)
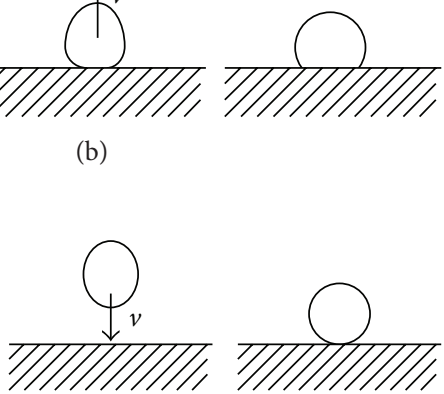

(c)

FIGURE 8: The sketch map of dynamic behaviors of droplet impacting on different solid surfaces.

that is, shrinking. However, as a result of different surface wettability, droplet motions on different solid surfaces show significant difference.

On the hydrophilic solid surface $\left(\mathrm{CA}=42.5^{\circ}\right.$, shown in Figure 4), the droplet spreads on the surface and dips into the microtopography gap quickly, forming the shape of flying saucer. Over time, the edge of the droplet gradually tilts, as the center portion becomes slightly thinner. The droplet changes into a disk shape. When the droplet spreads to its maximum, it begins to shrink under the action of surface tension and move toward the centerline, forming an upward flow, which will not depart from the surface owing to adhesion.

The droplet on the hydrophobic solid surface $(\mathrm{CA}=$ $119.3^{\circ}$, as shown in Figure 5) also experiences spreading and shrinking, but compared with motions on the hydrophilic surface, the droplet immerses into the gap only at the initial stage of contacting with the surface. In the shrinking process, fluid moves toward the center forming an upward flow. As the droplet is under stretching, the contact area of droplet and solid surface gradually decreases. Although the droplet has a tendency to depart, it cannot get away from the solid surface for having no sufficient kinetic energy to overcome the adhesion force.

For more hydrophobic solid surfaces $\left(\mathrm{CA}=138.2^{\circ}\right.$ and $\mathrm{CA}=151.8^{\circ}$, shown in Figures 6 and 7 , resp.), droplets also experience spreading and shrinking. However, droplets have not dipped into the gap, and they spread and shrink more quickly. In the shrinking process, fluid moves toward the center forming an upward flow. As droplets are under stretching, the contact area of droplet and solid surface decreases gradually until droplets can bounce up, away from the solid surface. Then droplets move along the horizontal and the vertical directions repeatedly. They rise in a manner of squirming, fall under the action of vertical force after reaching the highest point, and then experience another period of spreading and shrinking. It can be found by comparing Figures 6 and 7 that if the surface hydrophobicity is stronger, the maximum height of the droplet is greater, and the droplet will undergo more spreading and shrinking before reaching a steady state eventually.

Next, characteristics of dynamic behaviors of droplets impacting on solid surfaces will be analyzed from the view of energy. According to the principle of minimum energy, the stationary droplet on solid surfaces has a minimum surface energy. Droplets will go through two phases after hitting a solid surface: spreading and shrinking. The spreading behaviors of droplets on solid surfaces with different wettability are similar. Droplets with initial kinetic energy $E_{v}=m V_{0}^{2} / 2$ deviate from the center and move outward under inertia force against the adhesion force from solid surface, along with increase of the surface area and surface energy. In this phase, part of the kinetic energy of the droplet is converted into viscous dissipation energy $W$, and part of it is converted into surface energy $E_{s}$. When $E_{v}$ is running out, the droplet velocity drops to zero, while $E_{s}$ reaches maximum. Then the droplet shrinks inward under surface tension, with the surface area decreasing and the velocity increasing. Energy variation during the shrinking phase is to convert a portion of $E_{s}$ into kinetic energy $E_{v f}$ in order to overcome the acting of adhesion forces. If the rebound phenomenon occurs after the shrinking phase, it should happen along with the energy transition between kinetic energy and gravitational potential energy. So when the droplet is completely rebound, the minimum gravitational potential energy transferred from kinetic energy is $E_{h}=m g R$. Since the adhesive force to droplets on the hydrophobic surface is far less than that on the hydrophilic surface, the viscous dissipation is quiet small for the former droplets, which makes droplets still have a relatively high kinetic energy even after spreading and shrinking phases. This leads to the unique dynamic behavior of droplets on a hydrophobic surface:

(1) If the kinetic energy satisfies $E_{s f} \leq E_{v f}<E_{s}+E_{h}$ after the shrinking phase, the rebound phenomenon will not occur even for droplets with a tendency to pop up, as shown in Figure 8(b).

(2) With the hydrophobicity of solid surface enhanced, viscous dissipation during spreading and shrinking becomes smaller. If the kinetic energy satisfies $E_{v f} \geq$ $E_{s}+E_{h}$ after shrinking, droplets can move upward from the solid surface and the rebound phenomenon occurs, as shown in Figure 8(c). 


\section{Conclusion}

This paper simulates the wettability of hydrophobic surfaces and the dynamic behaviors of droplets impacting on hydrophobic surfaces with lattice Boltzmann method and gets the following conclusions:

(1) Lattice Boltzmann method is capable of considering both material properties and microtopography simultaneously, gives a relatively high precision, and shows great perspective in the study of the dynamic behaviors of droplets impacting on hydrophobic surfaces.

(2) The dynamic behaviors of droplets impacting on solid surfaces are affected by the wettability of the surface. The more hydrophobic the solid surface, the more likely the droplets rebound.

(3) If the droplet's kinetic energy is greater than the sum of surface energy and the minimum conversion gravitational potential energy when the spreading and shrinking finished, the rebound phenomenon will occur. As the hydrophobic surface's viscous dissipation is much smaller than hydrophilic surface's, the droplet still has high kinetic energy even after spreading and shrinking, which is advantageous to the rebound of droplets.

\section{Competing Interests}

The authors declare that they have no competing interests.

\section{Acknowledgments}

The work is supported by the National Natural Science Foundation of China (11502210, 51279165, and 51479170) and the Specialized Research Fund for the Doctoral Program of Higher Education of China (20126102110009).

\section{References}

[1] M. Nosonovsky and B. Bhushan, "Superhydrophobic surfaces and emerging applications: non-adhesion, energy, green engineering," Current Opinion in Colloid \& Interface Science, vol. 14, no. 4, pp. 270-280, 2009.

[2] J.-P. Zhao, X.-H. Shi, X.-G. Geng, and Z.-M. Hou, "Liquid slip over super-hydrophobic surface and its application in drag reduction," Journal of Ship Mechanics, vol. 13, no. 3, pp. 325-330, 2009.

[3] Z. L. Dou, J. D. Wang, F. Yu, and D. R. Chen, "Fabrication of a micro-structured surface based on interfacial convection for drag reduction," Fluid Mechanics, vol. 56, no. 7, pp. 626-632, 2011.

[4] M. R. Davidson, "Boundary integral prediction of the spreading of an inviscid drop impacting on a solid surface," Chemical Engineering Science, vol. 55, no. 6, pp. 1159-1170, 2000.

[5] M. Pasandideh-Fard, S. Chandra, and J. Mostaghimi, "A threedimensional model of droplet impact and solidification," International Journal of Heat and Mass Transfer, vol. 45, no. 11, pp. 2229-2242, 2002.

[6] H. Fujimoto, Y. Shiotani, A. Y. Tong, T. Hama, and H. Takuda, "Three-dimensional numerical analysis of the deformation behavior of droplets impinging onto a solid substrate," International Journal of Multiphase Flow, vol. 33, no. 3, pp. 317-332, 2007.

[7] Z. L. Guo and C. G. Zheng, Theory and Applications of Lattice Boltzmann Method, Science Press, Beijing, China, 2010.

[8] Y. L. He, Y. Wang, and Q. Li, Lattice Boltzmann Method: Theory and Applications, Science Press, Beijing, China, 2011.

[9] J. J. Huang, C. Shu, and Y. T. Chew, "Lattice Boltzmann study of droplet motion inside a grooved channel," Physics of Fluid, vol. 21, no. 2, Article ID 022103, pp. 1-11, 2009.

[10] Z. Y. Shi, G. H. Hu, and W. Zhou, "Lattice Boltzmann simulation of droplet motion driven by gradient of wettability," Acta Physica Sinica, vol. 59, no. 4, pp. 2595-2600, 2010.

[11] P. L. Bhatnagar, E. P. Gross, and M. Krook, "A model for collision processes in gases. I. Small amplitude processes in charged and neutral one-component systems," Physical Review, vol. 94, no. 3, pp. 511-525, 1954.

[12] X. W. Shan and H. D. Chen, "Lattice Boltzmann model for simulating flows with multiple phases and components," Physical Review E, vol. 47, no. 3, pp. 1815-1819, 1993.

[13] X. W. Shan and H. D. Chen, "Simulation of nonideal gases and liquid-gas phase transitions by the lattice Boltzmann equation," Physical Review E, vol. 49, no. 4, pp. 2941-2948, 1994. 


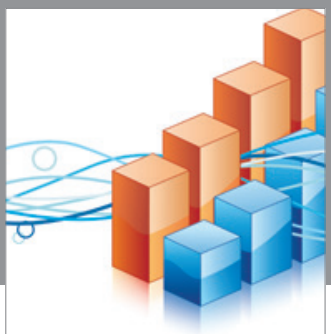

Advances in

Operations Research

vatem alat4

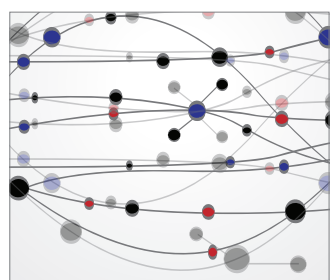

\section{The Scientific} World Journal
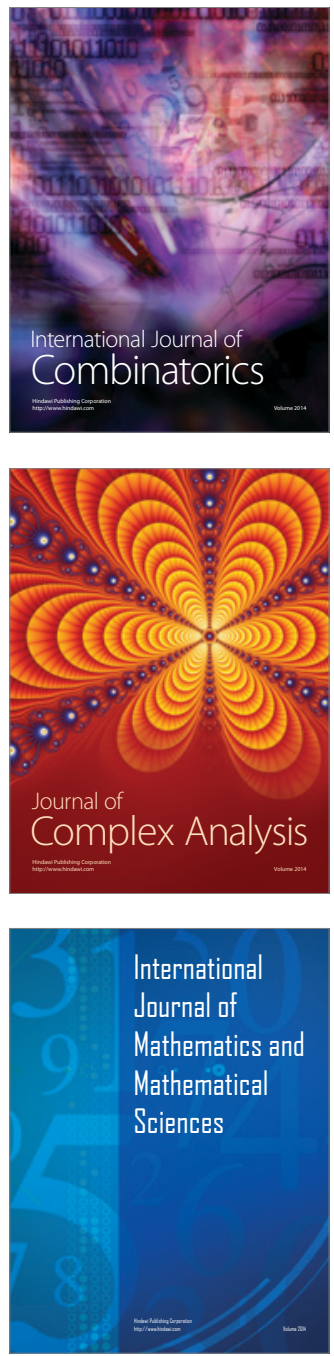
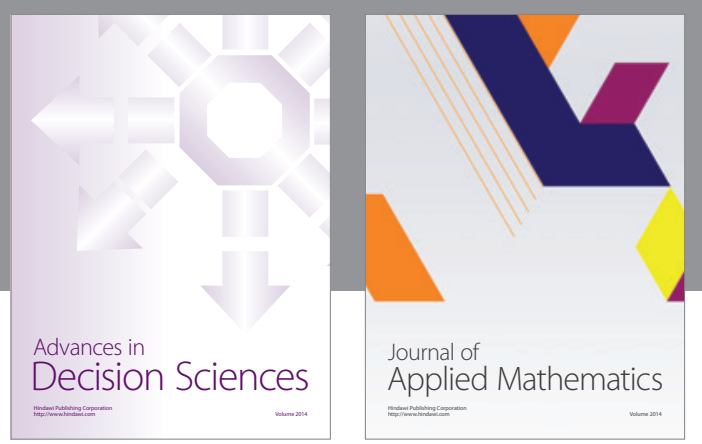

Algebra

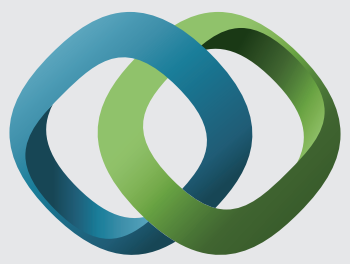

\section{Hindawi}

Submit your manuscripts at

http://www.hindawi.com
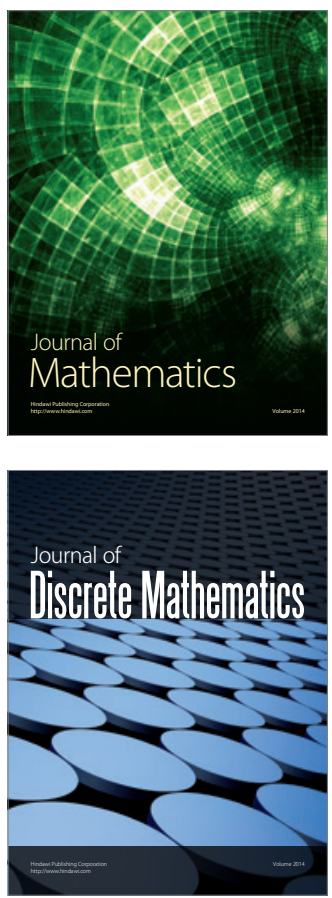

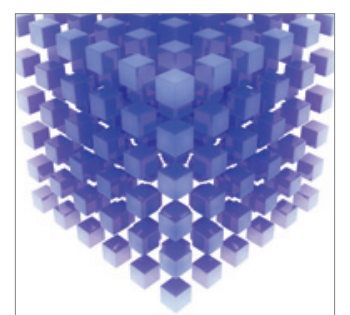

Mathematical Problems in Engineering
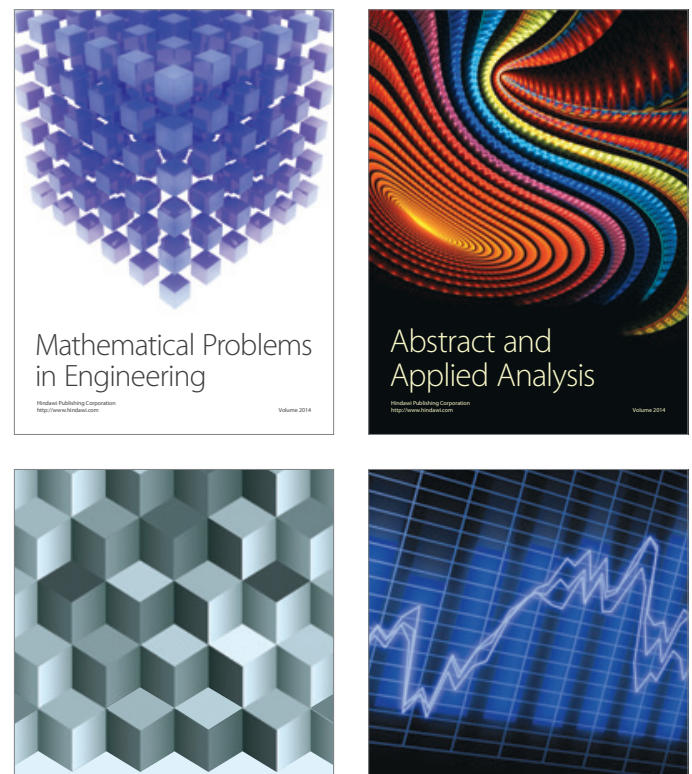

Journal of

Function Spaces

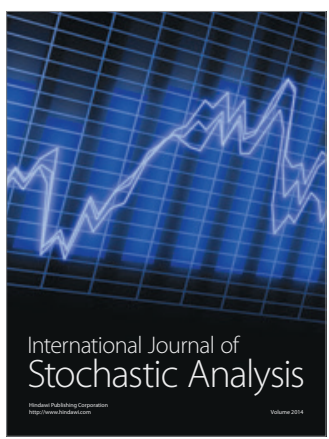

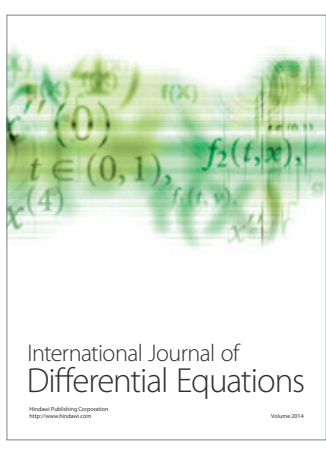
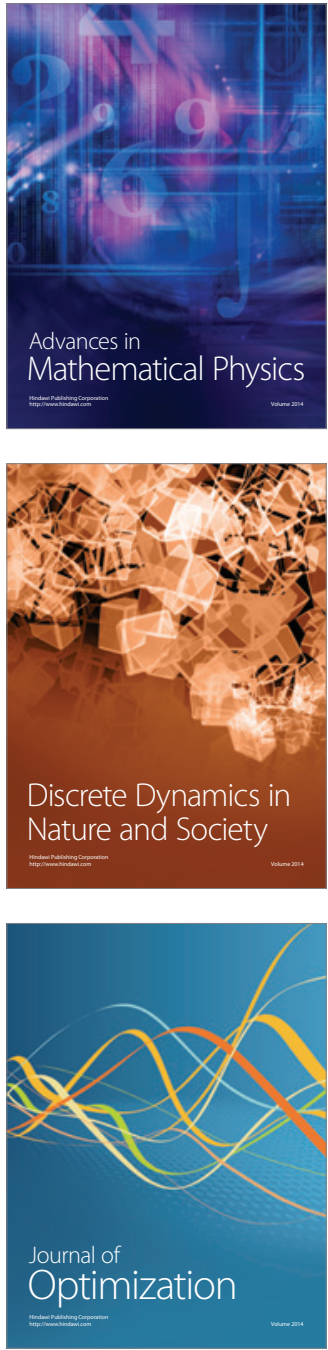